\title{
Coupling of structures using frequency response functions
}

\author{
Tiago Silva ${ }^{1, *}$ and João Pereira ${ }^{1}$ \\ ${ }^{1}$ NOVA UNIDEMI, Universidade NOVA de Lisboa, Campus de Caparica, 2829-516 Caparica, \\ Portugal.
}

\begin{abstract}
In the field of structural dynamics is common to predict the behaviour of a structure regarding structural modifications. In this context, the frequency based substructuring method is well-known to perform structural modifications based on the coupling of structures. This process gives the possibility to perform the study of a structure at the level of its components and then assess the response of the coupled system. In practice, it is impossible to attain an experimental complete response model, although one can simulate all the responses of a structure using numerical models. Hence, the substructuring process can be enhanced by the combined use of experimental and numerical responses, as it was demonstrated using numerically obtained frequency response functions. This work presents the enhancement of the frequency based substructuring method using a method to expand experimental frequency response functions over the entire set of degrees of freedom in a finite element model. This expansion process, known as modified Kidder's method, considers that if one can only measure translations due to exciting force, it is possible to obtain the complete response model, including the rotational frequency response functions due to exciting moments. The combined use of the frequency based substructuring and the modified Kidder's methods has several advantages, as it avoids modal identification or residual compensation. To evaluate the performance of the proposed procedure a numerical example of a beam structure is presented, and its results are discussed.
\end{abstract}

\section{Introduction}

Using structural modifications to predict the behaviour of a structure is a known practice in the field of structural dynamics. In this context, the frequency based substructuring (FBS) method [1] is a well-known method to perform structural modifications based on the coupling of substructures. This process gives the possibility to perform the study of a structure at the level of its components and then assess the response of the coupled system. To obtain a quality result in the coupling process, one should use a complete response model, which is experimentally unpractical or even impossible. However, one can simulate all the responses of a structure using numerical models. Usually, one can only measure few translation degrees of freedom (DoF) due to exciting forces. Hence, the rotational DoF are commonly disregarded in testing. On the other hand, if one considers the use of their numerical counter-parts, the

\footnotetext{
*e-mail: tan.silva@ fct.unl.pt
} 
substructuring process can be enhanced, as it was demonstrated using numerically obtained frequency response functions (FRF). An approach to tackle this problem was proposed by Drozg et al. [2].

This paper presents the enhancement of the FBS method using the modified Kidder's method [3] to expand a set of experimental FRFs over the entire set of DoFs in a finite element model. This expansion process may consider that one can only acquire responses at translational DoFs due to exciting force, and it enables to attain the complete response model, including the rotational FRFs due to exciting moments. The combined use of the FBS method and the modified Kidder's one has several advantages, as it avoids modal identification or residual compensation. To evaluate the performance of the proposed procedure a numerical example is presented, and its results are discussed. A beam structure is divided in two substructures and then used to simulate the pseudo-experimental FRFs of the complete structure and of its substructures. Note that only translational FRFs due to forces are considered to be available.

\section{Frequency based substructuring}

Considering a structure C, composed by two substructures A and B and considering the sets of DoFs given in Figure 1, it is possible write the complete receptance matrix of the structure $\mathrm{C}$ as,

$$
\mathbf{H}^{\mathrm{C}}(\omega)=\mathbf{H}^{\mathrm{C}}=\left[\begin{array}{lll}
\mathbf{H}_{i i} & \mathbf{H}_{i j} & \mathbf{H}_{i k} \\
\mathbf{H}_{j i} & \mathbf{H}_{j j} & \mathbf{H}_{j k} \\
\mathbf{H}_{k i} & \mathbf{H}_{k j} & \mathbf{H}_{k k}
\end{array}\right]
$$

The frequency dependence will be omitted for sake of simplicity. Note that DoFs $i$ are the ones in the domain of $\mathrm{A}$ and $k$ in $\mathrm{B}$. The DoFs $j$ denote DoFs shared by A and B in their interface. Hence, one has the receptance matrices of the substructures A and B given by,

$$
\mathbf{H}^{\mathrm{A}}=\left[\begin{array}{ll}
\mathbf{H}_{i i}^{\mathrm{A}} & \mathbf{H}_{i j}^{\mathrm{A}} \\
\mathbf{H}_{j i}^{\mathrm{A}} & \mathbf{H}_{j j}^{\mathrm{A}}
\end{array}\right] \quad \text { and } \quad \mathbf{H}^{\mathrm{B}}=\left[\begin{array}{cc}
\mathbf{H}_{k k}^{\mathrm{B}} & \mathbf{H}_{k j}^{\mathrm{B}} \\
\mathbf{H}_{j k}^{\mathrm{B}} & \mathbf{H}_{j j}^{\mathrm{B}}
\end{array}\right]
$$

regarding that, in the interface DoFs $j$, both the force equilibrium and the compatibility of displacements must be fulfilled, one has,

$$
f_{j}^{\mathrm{A}}+f_{j}^{\mathrm{B}}=f_{j}^{\mathrm{C}} \quad \text { and } \quad x_{j}^{\mathrm{A}}=x_{j}^{\mathrm{B}}=x_{j}^{\mathrm{C}}
$$

Therefore, eq. (1) can be recast, accordingly to the developed in [1], as,

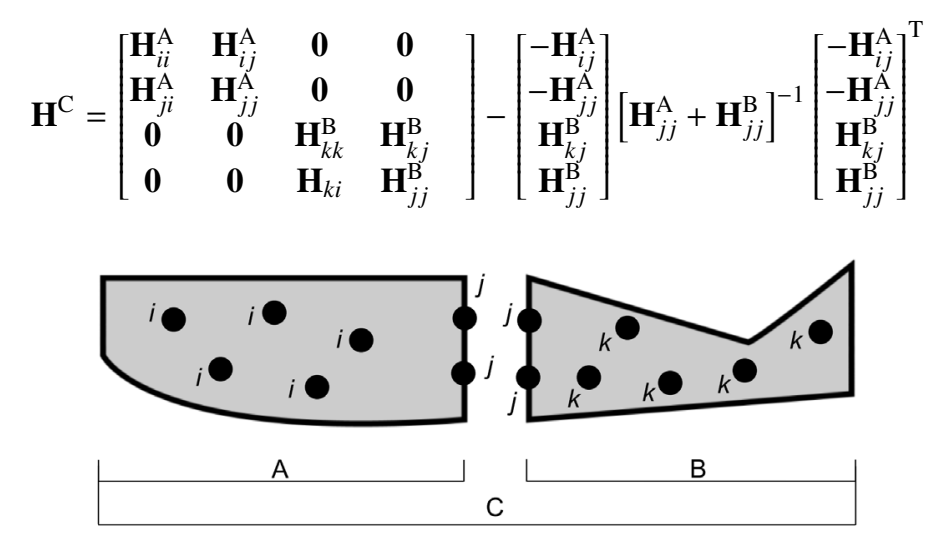
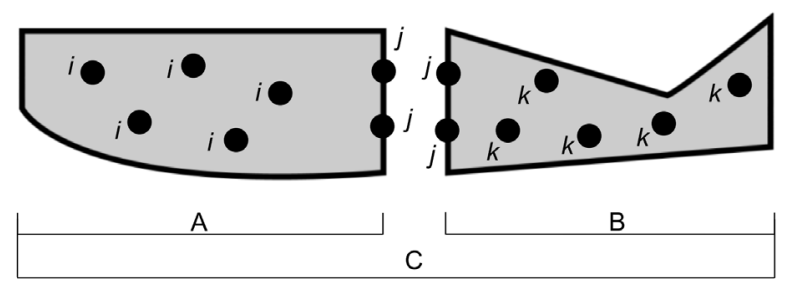

Figure 1: Schematic representation of a structure C, decoupled into two substructures A and B, with different sets of DoFs identified. 
From eq. (4), it can be seen that the receptance matrix of the complete structure $\mathrm{C}$ can be obtained from the knowledge of the receptance matrices of both substructures A and B. However, in practice, it is almost impossible to acquire data that allows to built those matrices completely, mainly due to difficulties with the excitation with pure moments or the ability to measure some DoFs, namely the rotational ones. Hence, one should consider an expansion technique suitable to expand a set of experimental FRFs over the receptance matrix elements that were not acquired. The use of the modified Kidder's method [3] is here discussed.

\section{Modified Kidder's method}

A modified version of the Kidder's method, applied in the frequency domain, is here considered to provide an expansion technique suitable to use experimental FRFs as they are collected.

The modified Kidder's method is derived from the partitioned dynamic equilibrium equation written as,

$$
\left(\left[\begin{array}{ll}
\mathbf{K}_{p p} & \mathbf{K}_{p s} \\
\mathbf{K}_{s p} & \mathbf{K}_{s s}
\end{array}\right]-\omega^{2}\left[\begin{array}{ll}
\mathbf{M}_{p p} & \mathbf{M}_{p s} \\
\mathbf{M}_{s p} & \mathbf{M}_{s s}
\end{array}\right]\right)\left\{\begin{array}{l}
\boldsymbol{H}_{p q}(\omega) \\
\boldsymbol{H}_{s q}(\omega)
\end{array}\right\}=\left\{\begin{array}{c}
\boldsymbol{I}_{q} \\
\mathbf{0}
\end{array}\right\}
$$

where the primary $p$ and secondary $s$ coordinates correspond to the measured and unmeasured DoFs, respectively, and $\boldsymbol{I}_{q}$ is a boolean vector mapping a given force location $q$.

From eq. (5) it can be shown that a FRF vector expanded for the unmeasured DoFs is given by,

$$
\boldsymbol{H}_{s j}(\omega)=-\left(\mathbf{K}_{s s}-\omega^{2} \mathbf{M}_{s s}\right)^{-1}\left(\mathbf{K}_{s p}-\omega^{2} \mathbf{M}_{s p}\right) \boldsymbol{H}_{p j}(\omega)
$$

The expansion process is here achieved spectral line by spectral line for a given experimental frequency range, for each acquired FRF vector $\boldsymbol{H}_{p j}(\omega)$.

In order to attain a complete FRF matrix from a set of measured FRFs, a two steps expansion strategy must be addressed. As given by Avitabile and O'Callahan [4] and adapted with the modified Kidder's method by Silva and Maia [3]. The expansion strategy relies on a careful definition of all the system DoFs, considering the specificities related to the response and excitation coordinates in the response model. Note that in a spatial model one has only primary and secondary coordinates, while in a response model the primary and secondary response coordinates have their dual in the excitation coordinate set. Therefore, consider a complete receptance matrix $\mathbf{H}$ partitioned wrt translational $t$ and rotational $\theta$ response DoFs and DoFs where force $f$ and moment $\tau$ excitations can be applied as,

$$
\mathbf{H}=\left[\begin{array}{ll}
\mathbf{H}_{t f} & \mathbf{H}_{t \tau} \\
\mathbf{H}_{\theta f} & \mathbf{H}_{\theta \tau}
\end{array}\right]
$$

where $t \equiv f$ and $\theta \equiv \tau$. Usually in practice, one can only measure a subset of $t$ and excite part of $f$, and often these subsets are not equal. Note that the possibility of measuring rotational DoFs or applying pure moments is here completely rejected, although they can be included. Hence, one must recast eq. (7) as,

$$
\mathbf{H}=\left[\begin{array}{cccc}
\mathbf{H}_{A A} & \mathbf{H}_{A E} & \mathbf{H}_{A U_{f}} & \mathbf{H}_{A \tau} \\
\mathbf{H}_{B A} & \mathbf{H}_{B E} & \mathbf{H}_{B U_{f}} & \mathbf{H}_{B \tau} \\
\mathbf{H}_{U_{t} A} & \mathbf{H}_{U_{t} E} & \mathbf{H}_{U_{t} U_{f}} & \mathbf{H}_{U_{t} \tau} \\
\mathbf{H}_{\theta A} & \mathbf{H}_{\theta E} & \mathbf{H}_{\theta U_{f}} & \mathbf{H}_{\theta \tau}
\end{array}\right]
$$

where the subsets of $t$ and $f$ type DoFs present in the FRF matrix can be organized by their nature, using the subscripts: $A$ for measured/forced DoFs, $B$ for measured/unforced DoFs, 


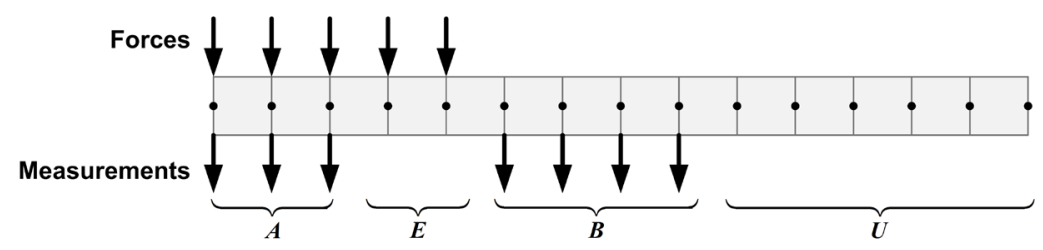

Figure 2: Illustration of the considered types of DoFs: $A$ measured/forced DoFs, $B$ measured/unforced DoFs, $E$ unmeasured/forced DoFs, $U$ unmeasured/unforced DoFs.

$E$ for unmeasured/forced DoFs, $U_{t}$ for unmeasured DoFs, and $U_{f}$ for unforced DoFs (see Figure 2). However, to ensure symmetry, one can map the unmeasured/forced DoFs $E$ onto the set of unmeasured DoFs $U_{t}$, reorganize $\mathbf{H}$ and recast eq. (8) as,

$$
\mathbf{H}=\left[\begin{array}{cccc}
\mathbf{H}_{A A} & \mathbf{H}_{A E} & \mathbf{H}_{A U_{f}} & \mathbf{H}_{A \tau} \\
\mathbf{H}_{B A} & \mathbf{H}_{B E} & \mathbf{H}_{B U_{f}} & \mathbf{H}_{B \tau} \\
\mathbf{H}_{E A} & \mathbf{H}_{E E} & \mathbf{H}_{E U_{f}} & \mathbf{H}_{E \tau} \\
\mathbf{H}_{U_{t} A} & \mathbf{H}_{U_{t} E} & \mathbf{H}_{U_{t} U_{f}} & \mathbf{H}_{U_{t} \tau} \\
\mathbf{H}_{\theta A} & \mathbf{H}_{\theta E} & \mathbf{H}_{\theta U_{f}} & \mathbf{H}_{\theta \tau}
\end{array}\right]=\left[\begin{array}{cccc}
\mathbf{H}_{A A} & \mathbf{H}_{A E} & \mathbf{H}_{A U_{f}} & \mathbf{H}_{A \tau} \\
\mathbf{H}_{E A} & \mathbf{H}_{E E} & \mathbf{H}_{E U_{f}} & \mathbf{H}_{E \tau} \\
\mathbf{H}_{U_{f} A} & \mathbf{H}_{U_{f} E} & \mathbf{H}_{U_{f} U_{f}} & \mathbf{H}_{U_{f} \tau} \\
\mathbf{H}_{\theta A} & \mathbf{H}_{\theta E} & \mathbf{H}_{\theta U_{f}} & \mathbf{H}_{\theta \tau}
\end{array}\right]
$$

Regarding the expansion of measured FRFs using the modified Kidder's method, it is possible to expand a set of measured FRFs avoiding the need of modal identification. Moreover, in terms of spectral incompleteness, the method is only limited by the acquired spectral lines and not by the number of identified modes, which eliminates residuals computation, and therefore their expansion.

Recalling eq. (8), in order to expand the measured FRFs over the entire set of model DoFs, one considers the expansion scheme here illustrated, using one of the relation given by eq. (6) and following the steps:

- expand the measured FRFs (the ones related to DoFs A, B and E) to obtain the FRFs at the unmeasured coordinates due to the applied excitation set, using the modified Kidder's method implementation;

- knowing all the FRFs at the set of forced DoFs, reorganize $\mathbf{H}$ as in eq. (9) and transpose the submatrices $\mathbf{H}_{\bullet A}$ and $\mathbf{H}_{\bullet E}$ to obtain $\mathbf{H}_{A \bullet}$ and $\mathbf{H}_{E \bullet}$;

- expand the transposed set of FRFs to obtain the remaining unknown part of $\mathbf{H}_{\bullet} U_{f}$ and $\mathbf{H}_{\bullet} \tau$.

\section{Numerical application: results and discussion}

As a numerical application, one has used a beam (structure $\mathrm{C}$ ) modelled by the finite element (FE) method, considering 49 2D beam elements with 2 DoFs per node (odd DoFs - translations; even DoFs - rotations). The beam (structure C) is $860 \mathrm{~mm}$ long with a rectangular cross-section with $16 \times 10 \mathrm{~mm}$, its modulus of elasticity is $210 \mathrm{GPa}$ and density $7680 \mathrm{~kg} / \mathrm{m}^{3}$. The substructures $\mathrm{A}$ and $\mathrm{B}$ are obtained by dividing $\mathrm{C}$ into its first $30 \mathrm{FE}$ and the remaining 19 , respectively. So, the node 31 is shared by both substructures.

To verify the results, Figure 3 shows FRFs of the complete structure obtained by the FE method and by the coupling of A and B using the FBS. Note that the match between FRFs is perfect as one considered the ability to know the entire receptance matrices of both substructures. Thus, the solution of the coupling problem is exact.

As expected, if one can acquire the complete FRF matrices for both substructures the coupling problem is easily tackled, even for rotational FRF due to an exciting moment $H_{20,40}^{\mathrm{C}}$. This kind of FRF is available in the FE model but it is not the case in common experimental 


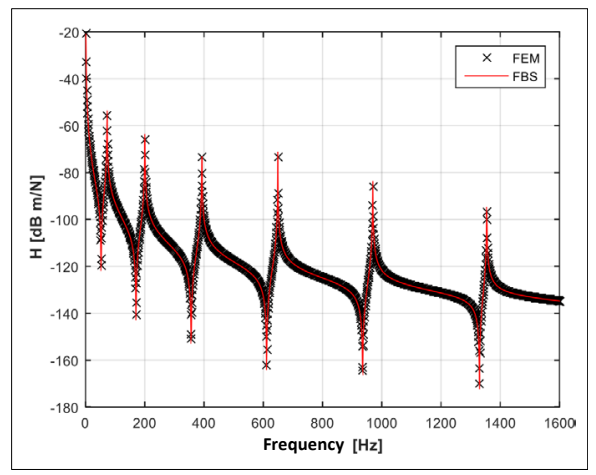

(a) $H_{5,1}^{\mathrm{C}}($ FRF type: $t f)$

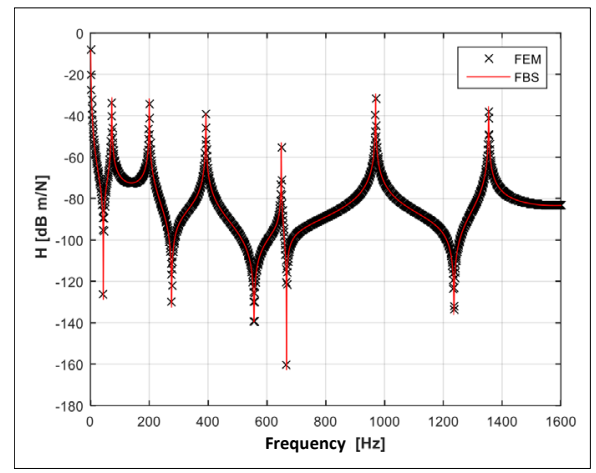

(b) $H_{20,40}^{\mathrm{C}}($ FRF type: $\theta \tau)$

Figure 3: Comparison of FRFs computed by the FBS method and the FE method, considering that the complete receptance matrices of both substructures A and B are available for the FBS method.

tests. Hence, the results in Figure 4 were obtained by the FBS, considering that only few translational due to exciting force FRFs are available from both substructures (A and B). So, the data used as acquired data is limited to the following sets of DoFs for the expansion process:

Substructure A: $A^{\mathrm{A}}=\left[\begin{array}{lll}3 & 913192327333943515761\end{array}\right] ; B^{\mathrm{A}}=E^{\mathrm{A}}=\emptyset$

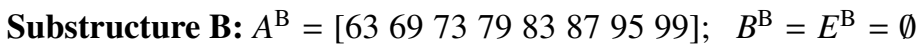

Note that all the FRFs in Figure 4 correspond to non-acquired FRFs, they were computed by the FBS method using the modified Kidder's method to expand the available data set.

From Figure 4, one can observe a quite good match between FRFs, especially in the frequency range bellow $600 \mathrm{~Hz}$. The deviation at higher frequencies is acceptable if compared to reference works, although it can be attenuated if more FRFs are known.

\section{Conclusion}

This paper presents the result of the combined implementation of the FBS method for coupling and the modified Kidder's method for the expansion of measured translational FRFs due to exciting forces. The expansion process is needed as the FBS method requires the complete receptance matrices of the substructures to be coupled and due to the fact that usually only translational due to exciting forces FRFs are experimentally acquired. A numerical example is used to demonstrate the capability of the proposed implementation and the obtained results show a high resemblance between the FRFs obtained with the FE method for the complete structure and the ones obtained with the coupling method, with incomplete receptance matrices. The experimental application of this approach has given also very good results, regarding the FRFs of the coupled structure. However, this case is out of the scope of this paper.

The authors acknowledge the support of the Portuguese Foundation for Science and Technology under the project UNIDEMI Pest-OE/EME/UI0667/2014.

\section{References}

[1] D. de Klerk, D.J. Rixen, J. de Jong, The frequency based substructuring (FBS) method reformulated according to the dual domain decomposition method, in IMAC-XXIV: Conference $\mathcal{E}$ Exposition on Structural Dynamics (SEM, 2006), ISBN 0912053941. 


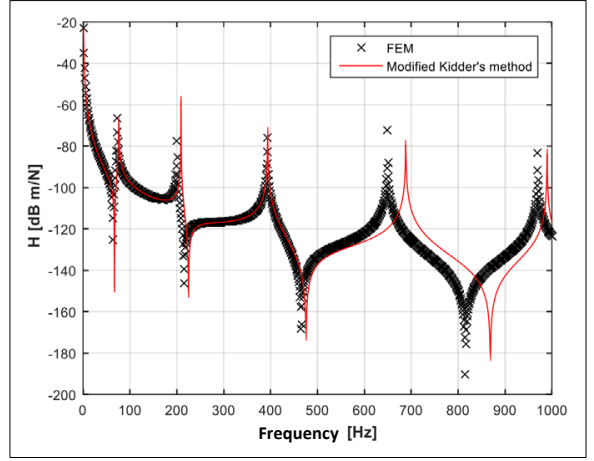

(a) $H_{3,17}^{\mathrm{C}}$ (FRF type: $t f$ )

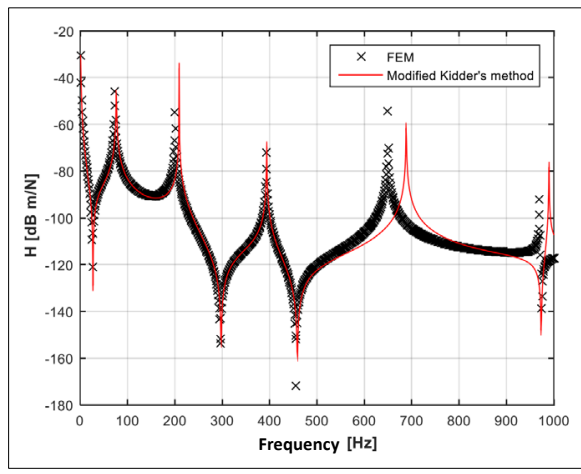

(c) $H_{80,41}^{\mathrm{C}}($ FRF type: $\theta f)$

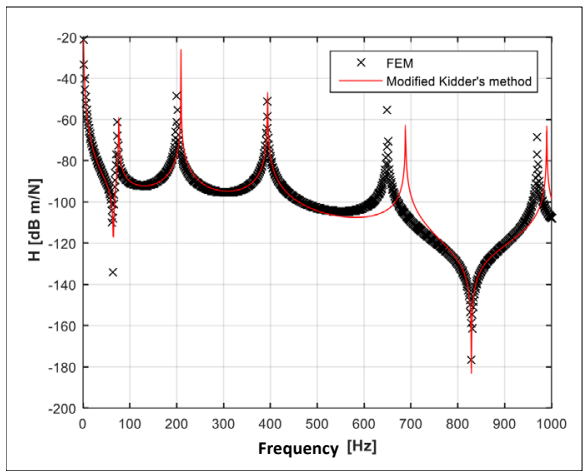

(b) $H_{25,90}^{\mathrm{C}}($ FRF type: $t \tau)$

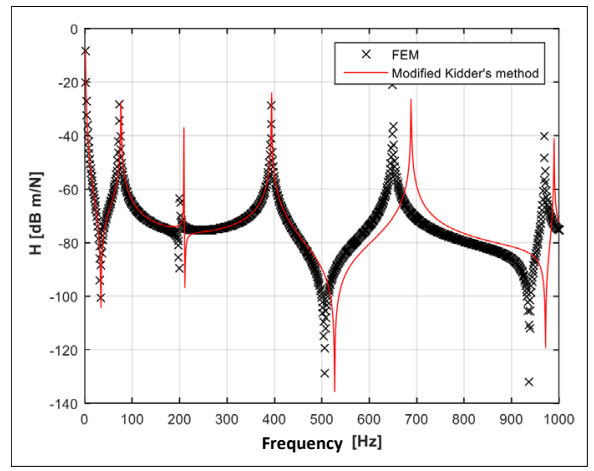

(d) $H_{32,6}^{\mathrm{C}}$ (FRF type: $\theta \tau$ )

Figure 4: Comparison of FRFs computed by the FBS method and the FE method, considering that few receptance vectors were acquired from substructures A and B.

[2] A. Drozg, G. Čepon, M. Boltežar, Mechanical Systems and Signal Processing 98, 570 (2018)

[3] T.A.N. Silva, N.M.M. Maia, in Conference Proceedings of the Society for Experimental Mechanics Series, edited by R.J. Allemang (Springer International Publishing, 2015), Vol. 6, pp. 35-48, ISBN 9783319069883.

[4] P. Avitabile, J. O'Callahan, Mechanical Systems and Signal Processing 17, 723 (2003) 